\title{
Saqueo y depredación de nidadas de tortugas marinas, durante las temporadas 2003 a 2012, en seis playas del Golfo de Paria, Venezuela
}

\author{
Clemente Balladares \& Eva Dubois \\ Oficina Nacional de Diversidad Biológica, Ministerio del Poder Popular para el Ambiente, Venezuela; cballadares@minamb.gob.ve
}

Recibido 21-II-2014 • Corregido 25-VI-2014 • Aceptado 31-VII-2014

\begin{abstract}
Poaching and predation of sea turtle clutches, during the 2003 and 2012 seasons, on six beaches of the Paria Gulf in Venezuela. Looting and predation of nests are among the major threats to marine turtles. Six beaches of Paria Gulf, northeastern Venezuela, have been protected and had a reduction of clutch poaching and predation on leatherback (Dermochelys coriacea), green (Chelonia mydas), and hawksbill (Eretmochelys imbricata) turtles. During ten years (2003-2012) of nesting seasons (March through October) the six beaches were observed for nesting, poaching and predation. The most frequent losses are caused by poaching, followed by the common fox (Cerdocyon thous), opossum (Didelphis marsupialis) and tamandua (Tamandua tetradactila). The most poached beach is Silvano, followed by Los Garzos (main nesting beach at the site) and Cerezo, which is more distant from the conservation team activity.
\end{abstract}

Key words: Conservation, Dermochelys coriacea, Chelonia mydas, Eretmochelys imbricata, poaching, predation, Paria Gulf.
RESUMEN: Seis playas, al sur del Golfo de Paria en el nororiente de Venezuela, se han protegido y han tenido una disminución en el saqueo y depredación de nidadas en la tortuga marina cardón (Dermochelys coriacea), en la verde (Chelonia mydas) y la carey (Eretmochelys imbricata). Durante diez años (2003-2012) en temporadas de desove (marzo a octubre) se observó en las seis playas las nidadas, saqueos y depredación. Las pérdidas mas frecuentes son debido al saqueo, seguido del zorro común (Cerdocyon thous), zarigüeya (Didelphis marsupialis) y el oso melero (Tamandua tetradactyla). La localidad más afectada es Silvano seguida de Los Garzos (principal sitio de anidamientos) y luego Cerezo, que es la más lejana al equipo de labores.

Palabras clave: Conservación, Dermochelys coriacea, Chelonia mydas, Eretmochelys imbricata, saqueo, depredación, Golfo de Paria.
Cuando las poblaciones de tortugas marinas se encuentran en riesgo, el saqueo y/o depredación de nidadas en sus playas de desove es considerado como una de las principales amenazas a nivel mundial (Lutcavage, Plotkin, Witherington \& Lutz, 1997; Campbell, 2003; Ceballos, 2004). Como ejemplos representativos de este problema se encuentra el caso en Playa Langosta de Costa Rica donde desova Dermochelys coriacea y en donde el saqueo alcanza el $91 \%$ en los meses tope (Chaves et al., 1996). Otro lugar con alta pérdida de huevos es la Isla Redang en Malasia, con pérdidas de más del $83 \%$ de las nidadas de carey Eretmochelys imbricata, causadas por diferentes depredadores como larvas de moscas, hormigas, cangrejos y lagartijas (Chan \& Liew, 1999). En la Isla Cousine de las Seychelles se han reportado pérdidas del $35 \%$ en el total de huevos de nidos de carey debido a la depredación por el cangrejo fantasma Ocypode cordimana (Hitchins, Bourquim \& Hitchins, 2004).
En lo que respecta a tortugas dulceacuícolas, el terecay Podocnemys unifilis en el rio Aguarico en Ecuador es saqueado por las comunidades en un 28\% (Caputo, Canestrelli \& Boittani, 2005). En Colombia las hormigas y pequeños mamíferos depredan los huevos de $P$. erytrocephala con un $33 \%$ y $12 \%$ respectivamente (Castaño, Galvis \& Molano, 2003).

A pesar de no tener una cuantificación, en las nidadas de tortugas marinas de Venezuela este fenómeno sucede tanto por la acción de zorros cangrejeros Procyon cancrivorus y el zorro común Cerdocyon thous, como también por humanos (Guada \& Sole, 2000). Aunado a ser ilegal, el consumo y la comercialización de esos huevos en todo el territorio nacional es una actividad que se mantiene de manera furtiva. El problema aumenta en los poblados costeros remotos donde el cumplimiento de leyes ambientales es más difícil de controlar. 
Hacia el noreste del país, en el Estado Sucre, se ubica el pueblo de Macuro en el Golfo de Paria. En cinco playas cercanas a ese pueblo: Los Garzos, Macurito, Silvano, Obispo y Cerezo, y en ocasiones también en el litoral del mismo pueblo desovan más de un centenar de hembras de tres especies de tortugas marinas: Dermochelys coriacea, Chelonia mydas y Eretmochelys imbricata (Balladares, Quijada, Cova, Mora \& Dubois, 2009). Las nidadas de las dos especies de tortugas más abundantes: $D$. coriacea y E. imbricata, vienen siendo depredados por fauna silvestre y saqueados por humanos residentes en la zona golfo. Desde el año 2003 la Oficina Nacional de Diversidad Biológica (ONDB) del Ministerio del Ambiente de Venezuela lleva a cabo un Programa de Conservación de Tortugas Marinas. Gracias a este esfuerzo se ha logrado disminuir el consumo de huevos. En el presente artículo se muestran algunos valores que reflejan el control sobre los depredadores debido a la presencia continua de un equipo de trabajo durante diez temporadas de desove en la región.

\section{MATERIALES Y MÉTODOS}

Área de estudio: La península y el Golfo de Paria están ubicados en el extremo nororiental de Venezuela, en el estado Sucre. En ese lugar, personal del Programa de Conservación de Tortugas Marinas que adelanta la Oficina Nacional de Diversidad Biológica (ONDB) recorre seis playas desde el año 2003 y a partir de 2008 se lleva un control más regular en las playas: Los Garzos, Silvano, Macurito, Obispo, Macuro y Cerezo.

Estas playas tienen longitudes de costa menores a $300 \mathrm{~m}$, teniendo la mayoría un promedio de $150 \mathrm{~m}$ de sustrato arenoso y unos $5 \mathrm{~m}$ de ancho. El resto de la superficie es pedregosa y en la mayoría de ellas se observa una franja supra litoral de vegetación de manzanillo (Hippomane mancinella) y cremón (Thespesia popuinae).

Trabajo de campo: Entre los años 2003 y 2012 se registraron las anidaciones que pudieron ser detectadas, así como los saqueos y/o depredaciones ocurridos, asociando el tipo de depredador como el más probable, según las evidencias observadas y fotografiadas en las seis playas citadas anteriormente. Inicialmente durante los primeros cinco años se realizó un estimado general contabilizando solamente el número de las nidadas saqueadas y/o depredadas por total de playas, sin discriminar los tipos ni características del patrón de saqueo.

Desde marzo hasta octubre, de cada año, se hacen recorridos inter-diarios en las diferentes playas, únicamente se pernocta y se recorre detalladamente en Los Garzos desde las $8 \mathrm{pm}$ hasta las $3 a \mathrm{~m}$ ya que es donde ocurre el $65 \%$ de los desoves y donde se tiene un campamento adecuado. La mayoría de las nidadas son trasplantadas a un vivero en el pueblo de Macuro en la medida de las posibilidades de traslado. A partir de 2008 , en cada nidada perturbada se hizo un registro de la playa, la especie de tortuga marina, el tipo de perturbación (saqueada o depredada) y la especie depredadora, además de un registro fotográfico.

\section{RESULTADOS}

Desde el 2003 hasta el 2012 se registro un total de 950 desoves entre Eretmochelys imbricata, Dermochelis coriacea y Chelonia mydas, con un promedio de 110,7 nidadas por año (Fig. 1). Del total de nidadas el 22,84\% fue saqueado y depredado (Fig. 2), pero se observa una tendencia sostenida a disminuir la tasa de perdida. Durante el 2003 se contabilizaron 42 nidos. La presencia del equipo de trabajo formado localmente en la zona en 2005, más el aumento de las salidas de campo permitió registrar la pérdida de sólo siete nidadas en 2008 , con un aumento a un máximo de 20 en años posteriores.

El porcentaje del saqueo y depredación se cálculo año a año como un índice del daño ocasionado por los depredadores (Fig. 3). En la gráfica se observa con mayor detalle el daño que hace la depredación de nidadas sobre la reproducción de las tortugas marinas en la zona

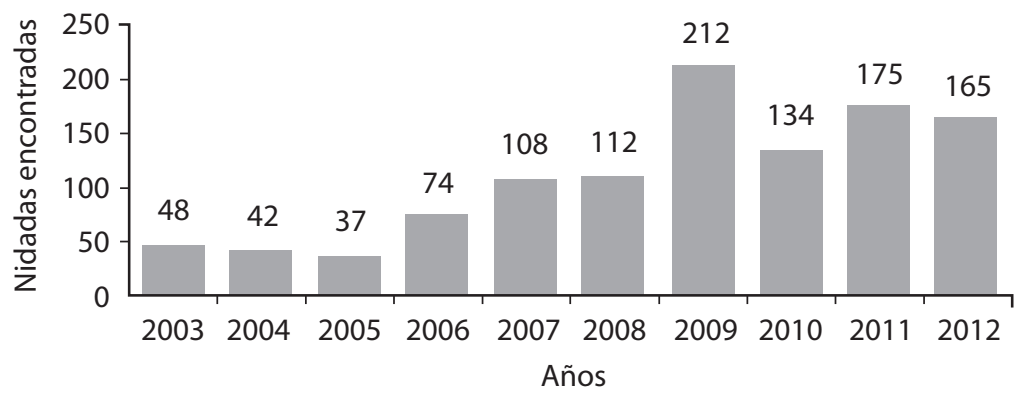

Fig. 1. Total de nidadas registradas de Tortugas Marinas en el Golfo de Paria durante las temporadas 2003-2012. 


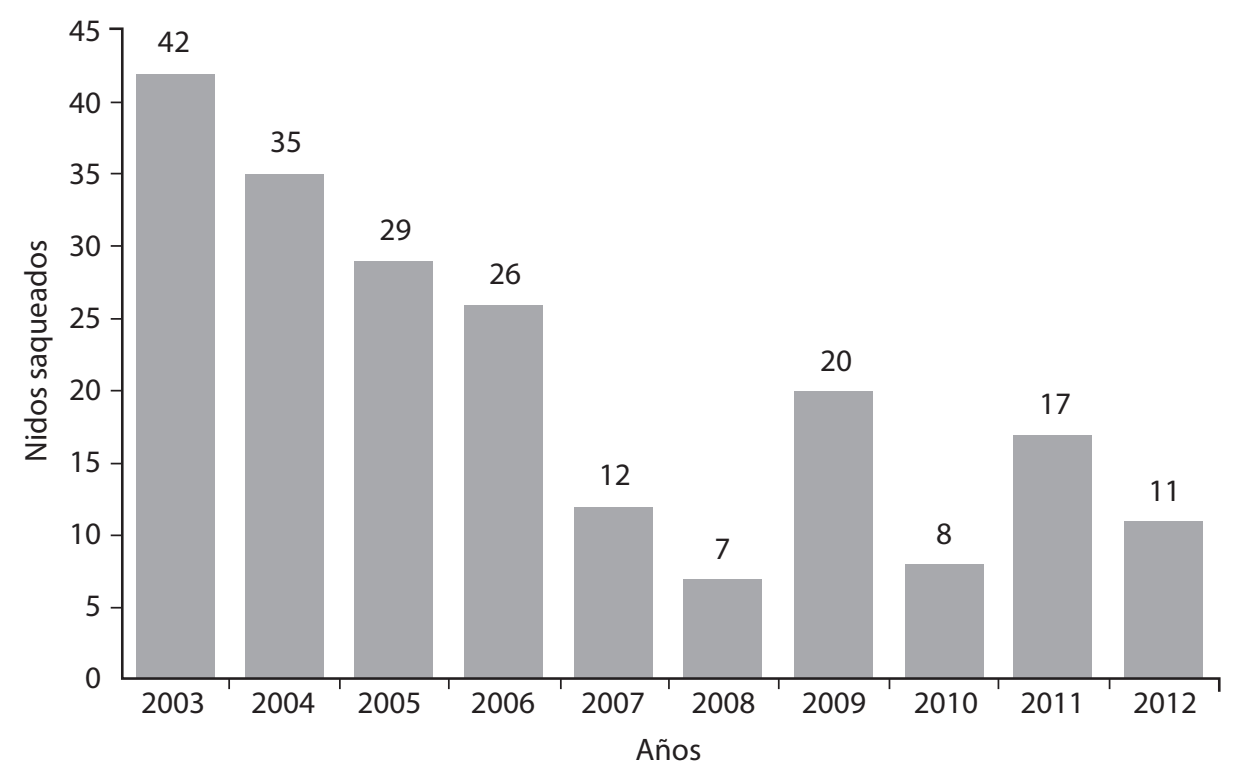

Fig. 2. Número de nidadas perdidas por saqueos y/o depredación por año desde 2003 a 2012.

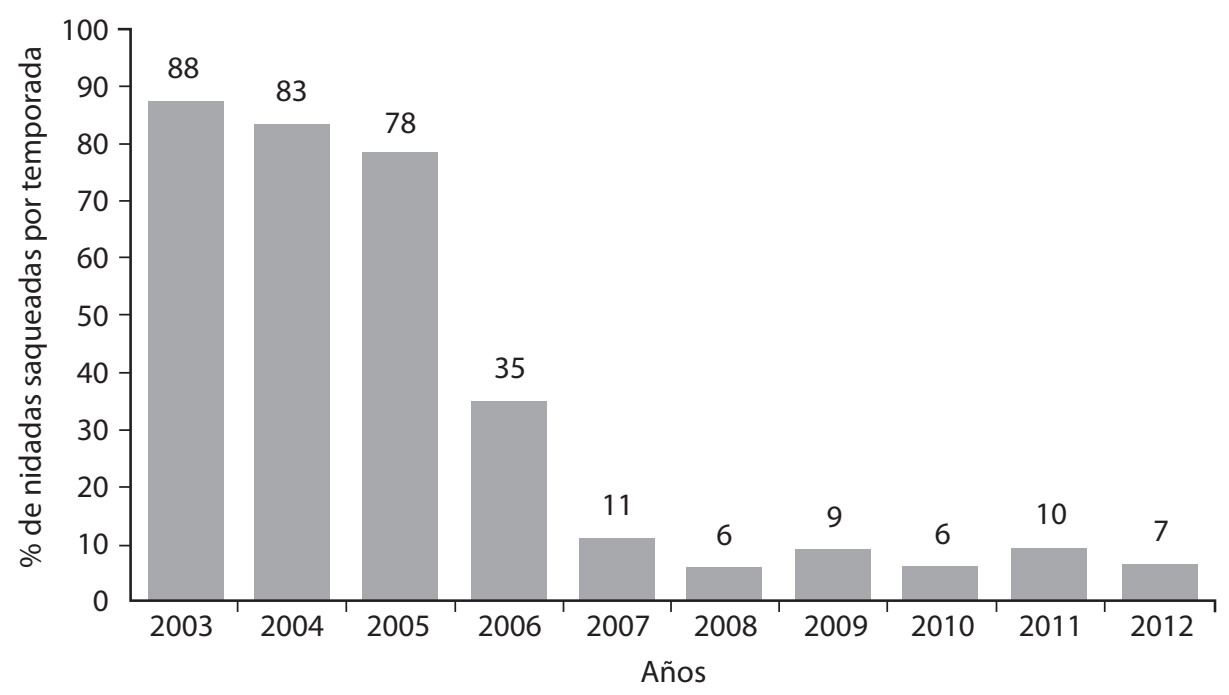

Fig. 3. Porcentaje de nidadas perdidas por saqueo y/o depredación por temporada (2003 a 2012).

de estudio durante la primera mitad de la década que se inicio con un $87,5 \%$ de nidos saqueados y depredados. Al contrario la disminución del porcentaje hasta un mínimo de 6,25\% a partir del quinto año -2007- refleja el éxito de conservación por la presencia del equipo local de labores.

A partir del año 2008 hasta la temporada 2012 se comenzó a trabajar de manera más permanente con un equipo residente y detallando mejor la afectación de saqueos por especie (cardón, verde o carey, Cuadro 1).
De estas tres especies, Eretmochelys imbricata es la más perjudicada por los saqueos y depredación.

La playa más afectada por la pérdida de nidadas desde el año 2008 es Silvano con 18 nidos, seguido por Los Garzos (17), y luego Cerezo (15) (Cuadro 1). Macurito y Obispo fueron las localidades con menor perdida (7 y 6 respectivamente). En el pueblo de Macuro no se reportaron nidadas afectadas.

El tipo de depredador que perturba más los desoves en la zona de estudio es el humano quien siempre 
CUADRO 1

Número de nidadas saqueadas y depredadas por especie y playa, por año

\begin{tabular}{|c|c|c|c|c|c|c|c|c|c|c|c|c|c|c|c|c|c|c|c|c|c|c|c|c|c|c|c|c|c|c|}
\hline \multirow{2}{*}{$\begin{array}{l}\text { Localidad } \\
\text { Especie }\end{array}$} & \multicolumn{6}{|c|}{ Los Garzos } & \multicolumn{6}{|c|}{ Silvano } & \multicolumn{6}{|c|}{ Obispo } & \multicolumn{6}{|c|}{ Macurito } & \multicolumn{6}{|c|}{ Cerezo } \\
\hline & \multicolumn{2}{|c|}{$D c$} & \multicolumn{2}{|c|}{$E i$} & \multicolumn{2}{|c|}{$\mathrm{Cm}$} & \multicolumn{2}{|c|}{$D c$} & \multicolumn{2}{|c|}{$E i$} & \multicolumn{2}{|c|}{$\mathrm{Cm}$} & \multicolumn{2}{|c|}{$D c$} & \multicolumn{2}{|c|}{$E i$} & \multicolumn{2}{|c|}{$\mathrm{Cm}$} & \multicolumn{2}{|c|}{$D c$} & \multicolumn{2}{|c|}{$E i$} & \multicolumn{2}{|c|}{$\mathrm{Cm}$} & \multicolumn{2}{|c|}{$D c$} & \multicolumn{2}{|c|}{$\mathrm{Ei}$} & \multicolumn{2}{|c|}{$\mathrm{Cm}$} \\
\hline Causa & $\mathbf{S}$ & D & $\mathbf{S}$ & D & $S$ & D & $\mathbf{S}$ & D & $\mathbf{S}$ & D & $\mathbf{S}$ & D & $\mathbf{S}$ & D & S & D & $\mathbf{S}$ & D & $\mathbf{S}$ & D & $\mathbf{S}$ & D & $\mathbf{S}$ & D & $S$ & D & $\mathbf{S}$ & D & $\mathbf{S}$ & D \\
\hline 2008 & 0 & 2 & 2 & 3 & 0 & 0 & 0 & 0 & 0 & 0 & 0 & 0 & 0 & 0 & 0 & 0 & 0 & 0 & 0 & 0 & 0 & 0 & 0 & 0 & 0 & 0 & 0 & 0 & 0 & 0 \\
\hline 2009 & 0 & 1 & 1 & 1 & 0 & 0 & 0 & 2 & 2 & 1 & 0 & 0 & 0 & 0 & 1 & 1 & 0 & 0 & 0 & 0 & 0 & 0 & 0 & 0 & 0 & 0 & 8 & 2 & 0 & 0 \\
\hline 2010 & 0 & 0 & 0 & 0 & 0 & 0 & 0 & 0 & 4 & 2 & 1 & 1 & 0 & 0 & 0 & 0 & 0 & 0 & 0 & 0 & 0 & 0 & 0 & 0 & 0 & 0 & 0 & 0 & 0 & 0 \\
\hline 2011 & 0 & 0 & 4 & 3 & 0 & 0 & 1 & 0 & 2 & 0 & 0 & 0 & 0 & 0 & 0 & 2 & 0 & 0 & 1 & 0 & 0 & 0 & 0 & 0 & 0 & 0 & 4 & 0 & 0 & 0 \\
\hline 2012 & 0 & 0 & 0 & 0 & 0 & 0 & 0 & 0 & 2 & 0 & 0 & 0 & 0 & 0 & 0 & 2 & 0 & 0 & 0 & 0 & 4 & 2 & 0 & 0 & 0 & 0 & 1 & 0 & 0 & 0 \\
\hline TOTAL & 0 & 3 & 7 & 7 & 0 & 0 & 1 & 2 & 10 & 3 & 1 & 1 & 0 & 0 & 1 & 5 & 0 & 0 & 1 & 0 & 4 & 2 & 0 & 0 & 0 & 0 & 13 & 2 & 0 & 0 \\
\hline
\end{tabular}

$\mathrm{Dc}=$ Dermochelis coriácea, Ei=Eretmochelys imbricata, $\mathrm{Cm}=$ Chelonia mydas, $\mathrm{S}=$ saqueado, $\mathrm{D}=$ depredado.

saquea la totalidad de huevos por nidada. Los mamíferos pequeños observados directamente -Fotos anexas- o por evidencia de su actividad, (huellas y heces) son principalmente el zorro común (Cerdocyon thous) y otros como el rabopelado (Didelphis marsupialis) y el oso melero (Tamandua tetradactyla). Estos depredadores no consumen la totalidad de la nidada, por lo que en estos casos la depredación generalmente es parcial.

Pocos de estos depredadores fueron observados directamente, sin embargo, las huellas dejadas son conspicuas. Los cazadores furtivos se llevan toda la nidada, dejando un hueco bastante profundo y las marcas de los pies desnudos o en calzado en los alrededores. Los zorros destacan por consumir entre la mitad o el $75 \%$ de los huevos cavando someramente y en grupos de dos o más individuos, y si son sorprendidos en la actividad huyen rápidamente. El rabopelado actúa en solitario, las marcas de sus patas son mayores y consumen casi la mitad de los huevos por nidada. El oso melero camina esquivo, es más lento para escapar si es detectado y únicamente rompe menos de un tercio de la nidada lamiendo los huevos. En este último punto no se conocen referencias para T. tetradactyla como depredador de huevos de tortugas marinas.

\section{DISCUSIÓN}

De los resultados obtenidos en una década de labores en el Golfo de Paria es evidente la acción benéfica sobre la supervivencia de las nidadas debido a la presencia en la zona del equipo de conservación, repercutiendo en la disminución de los saqueos y/o depredación de nidadas de tortugas marinas. Otros programas de conservación con estas especies de fauna lograron un éxito similar en Venezuela, siendo el caso emblemático el de Isla de Aves donde se ha comprobado el aumento poblacional desde $373 \pm 12,5$ de hembras de Chelonia mydas en 2001 a $669 \pm 56,1$ para 2008 , gracias al establecimiento en el lugar de una estación naval en 1978 y a la presencia constante de un equipo de trabajo, por lo que no es un área de extracción de huevos (Vera \& Buitrago, 2012). Esta remota isla ha sido ejemplo de conservación eficiente durante los últimos 36 años. El éxito de este manejo adecuado es también reflejado en México por García, Ceballos y Adaya (2003) donde pérdidas de más del $90 \%$ por saqueo y depredación de coatíes (Nasua narica) fueron reducidas a menos del $10 \%$ debido al establecimiento de personal bien entrenado para cuidar los anidamientos de Lepidochelys olivacea en playa Cuixmala.

Específicamente hablando de cuantificación de saqueos de nidadas, en nuestro país no había estimaciones precisas de este fenómeno. Comparando con datos de Costa Rica para Dermochelys coriacea, se registraron valores tan altos como el $90,7 \%$ de saqueos cuando se carecía del patrullaje en las playas de desove, mientras que el porcentaje de saqueos disminuyó a 50,2\% cuando los grupos de conservación hicieron presencia (Chaves et al., 1996). En lo referente a la fauna silvestre depredadora, el daño a los huevos de Eretmochelys imbricata por el consumo de cangrejos y lagartijas en una isla de Malasia alcanza hasta el 83\% del total de nidos (Chan \& Lew, 1999).

De las seis playas protegidas y manejadas durante la década referida y con mayor énfasis desde 2008, playa Silvano nunca ha tenido presencia permanente de nuestro equipo, además de ser una playa cubierta bajo abundante vegetación litoral que puede guarecer a los depredadores, tanto humanos como pequeños mamíferos y con buena disponibilidad de nidadas de las tres especies de quelonios. Quizás por esto, se puede inferir que es la más comprometida en lo referente a la pérdida de nidadas. La principal área de desove es playa Los Garzos con un $65 \%$ del total de anidamientos, siendo 
también la de mayor presencia por el equipo de conservación. Sin embargo, Los Garzos está en segundo lugar de afectación. La playa de Cerezo, aunque es la que presenta la menor cantidad de desoves, también es una localidad muy afectada debido a su lejanía para el grupo de protección.

La preferencia de saqueos por especie en nuestro estudio, demostró que los huevos de $D$. coriacea son menos consumidos tanto por los pequeños mamíferos como por los húmanos. Conversaciones coloquiales con lugareños indican que los huevos de cardón, a pesar de su gran tamaño, son muy grasosos y de menos gusto. La tortuga verde Chelonia mydas es poco afectada por su bajo número de desoves (ocho en una década). Sin embargo, los dos saqueos parciales registrados representan la cuarta parte de esos anidamientos. Sin embargo, en términos concretos $E$. imbricata es la especie más perjudicada tanto por la abundancia de desoves -que la hacen más disponible- como por la presión de consumo por los humanos que representan el $67 \%$ de las pérdidas, a lo cual se suma los de la fauna local con un $25 \%$ del total.

Como reflexión final hay que considerar que, aparte de las leyes de protección, nacionales como internacionales, existe la posibilidad de explotar un recurso dependiendo de su disponibilidad y necesidad del ser humano. No obstante, en el caso de este estudio ello no es posible ya que los resultados indican que si se mantuvieran los niveles de depredación y sobre todo el saqueo, la supervivencia de esta población estaría gravemente comprometida. Mantener este programa en nuestra particular circunstancia regional, es vital para asegurar la conservación de las nidadas de tortugas marinas y es fundamental para la supervivencia de estas especies, al menos en el ámbito del Golfo de Paria. Hay que redoblar esfuerzos para mantener bajos los índices de saqueo y depredación de estos animales, a fin de incrementar a largo plazo el aporte reproductivo para la generación de relevo.

\section{AGRADECIMIENTOS}

A la Oficina Nacional de Diversidad Biológica del Ministerio del Ambiente, al Instituto Nacional de Parques, ConocoPhillips, PetroSucre y a PDVSA Costa Afuera junto a sus contratistas en la región: AmbioConsult, Z\&P, CAMUDOCA y Neptunia,C.A. por todos los recursos para el Programa de Conservación de Tortugas Marinas en el Golfo de Paria. A todos los asistentes de campo, voluntarios y macureños por el esfuerzo en las playas, a Ricardo Babarro y Aurora Sanz por la ayuda en análisis de datos y elaboración de las gráficas, a José Ramón Delgado, Eneida Marín y José Alió por la lectura crítica. Al amigo
Luis Cova por su siempre útil compañía, sugerencias y las fotografías. Finalmente en memoria del Biólogo Joaquín Buitrago por sus siempre útiles comentarios.

\section{REFERENCIAS}

Balladares, C., Quijada, A., Cova, L., Mora, B., \& Dubois, E. (2009). Conservación de tortugas marinas en el Golfo de Paria. Resúmenes del VIII Congr. Venezolano de Ecología.

Campbell, L. (2003). Contemporary culture, use, and conservation of Sea Turtles. In P. Lutz, J., Muzick \& J., Wyneken (Eds). The Biology of Sea Turtles (pp. 307-338). FI, EEUU: CRC press.

Caputo, F., Canestrelli, D., \& Boittani, L. (2005). Conserving the Terecay (Podocnemys unifilis, Testudines: Pelomudisidae) through a community-based sustainable harvest of its eggs. Biology Conservation, 126, 84-92.

Castaño-Mora, O., Galvis-Peñuela, P., \& Molano, J. G. (2003). Reproductive ecology of Podocnemys erytrocephala (Testudines: Podocnemididae), in the lower Inirida River in Colombia. Chelonian Conservation Biology, 4(3), 664-670.

Ceballos, C. (2004). Distribución de playas de anidación y áreas de alimentación de tortugas marinas y sus amenazas en el Caribe Colombiano. Boletín Investigación Marina Costera, 33, 79-99.

Chan, E., \& Liew, H. (1999). Hawksbill turtles, Eretmochelys imbricata, nesting on Redang Island, Terengannu, Malaysia, from 1993 to 1997. Chelonian Conservation Biology, 3(2), 326-329.

Chaves, A., Serrano, G., Marin, G., Arguedas, E., Jiménez, A., \& Spotila, J. (1996). Biology and conservation of leatherback turtles, Dermochelys coriacea, at Playa Langosta, Costa Rica. Chelonian Conservation Biology, 2(2), 184-189.

García, A., Ceballos, G., \& Adaya, R. (2003). Intensive beach management as an improved sea turtle conservation strategy in Mexico. Biological Conservation, 111, 253-261.

Guada, H., \& Sole, G. (2000). Plan de acción para la recuperación de las tortugas marinas de Venezuela. WideCast Inf. Tec, 39, 1-114.

Hitchins, P., Bourquim, O., \& Hitchins, S. (2004). Nesting success of hawksbill turtle (Eretmochelys imbricata) on Cousine Island, Seychelles. Journal of Zoology, 264, 393-389.

Lutcavage, M; Plotkin, P., Witherington, B., \& Lutz, P. (1997). Human impact on sea turtle survival. En P., Lutz \& J. Muzick (Eds.), The Biology of Sea Turtles (pp. 387-404). Fl, EEUU: CRC press.

Vera, V., \& Buitrago, J. (2012). Actividad reproductiva de Chelonia mydas (Testudines: Chelonidae) en Isla de Aves, Venezuela (2001-2008). Revista de Biología Tropical, 60 (2), 745-758. 
